\title{
Editorial
}

\author{
Weili Lin • William J. Powers
}

Received: 30 March 2012 /Accepted: 3 April 2012 /Published online: 17 April 2012

(C) Springer Science+Business Media, LLC 2012

Non-invasive imaging approaches, including computed tomography (CT), magnetic resonance imaging (MRI), and positron emission tomography (PET), have been employed extensively in the study of cerebral ischemia. With the exception of non-contrast CT, which is of proven value for identifying acute stroke patients who will benefit from intravenous tissue plasminogen activator (IV-tPA), imaging approaches had largely been considered research tools. While PET is capable of providing unique physiological measurements of cerebral hemodynamics and the cerebral metabolic rate of oxygen utilization [1-3], the logistics of using PET in an acute setting have largely hampered investigations of its clinical utility. On the other hand, MR is readily available and applicable to most patients with acute stroke. Diffusion-weighted imaging (DWI) reveals the anatomical locations of ischemic lesions soon after onset [4]. Relative measures of cerebral hemodynamics are also available with MR perfusion-weighted imaging (PWI) [5]. Given these capabilities and the wide availability of MRI, one could have expected that MR would have been shown to

\section{W. Lin}

Department of Radiology,

University of North Carolina at Chapel Hill,

Chapel Hill, NC, USA

W. Lin $(\bowtie)$

Biomedical Research Imaging Center,

University of North Carolina at Chapel Hill,

CB\#7513,

Chapel Hill, NC 27599, USA

e-mail: weili_lin@med.unc.edu

\section{W. J. Powers}

Department of Neurology,

University of North Carolina at Chapel Hill,

Chapel Hill, NC, USA be of critical importance for the management of acute stroke patients. Practically, however, this has not been the case.

Since the approval of tPA [6-8], extensive efforts have been devoted attempting to extend its therapeutic window based on the recognition that the current practice of "timeto-treat" may be sub-optimal and could be replaced with "tissue-to-treat." Numerous publications have suggested the existence of salvageable tissue in a sub-group of patients beyond the approved therapeutic window of tPA $[9,10]$. That is, if tPA were given to this sub-group of patients beyond the approved time window, it would lead to favorable clinical outcome, particularly if reperfusion is accomplished. Thus, a means to identify this sub-group of patients in a timely manner could extend the clinical benefit of tPA beyond $4.5 \mathrm{~h}$. More recently, endovascular clot retrieval devices have promised to further improve the efficacy of reperfusion, and three devices (MERCI, Penumbra, and Solitaire) have already received FDA approval [11-13]. However, with the logistics of utilizing invasive endovascular clot retrieval devices in patients and their accompanying risk of symptomatic intracranial hemorrhage, a means to identify a sub-group of patients who will benefit from these invasive procedures is of critical importance. Therefore, there is an increasing desire to utilize non-invasive imaging approaches for potentially identifying a sub-group of patients who may benefit from reperfusion therapies beyond the currently approved therapeutic window $(4.5 \mathrm{~h})$. Toward this end, the diffusion/perfusion mismatch concept has been widely advocated as a potential approach to identify such patients [14-16]. The underlying hypothesis is that lesions defined by abnormal diffusion most likely reflect irreversible injury while regions defined by abnormal perfusion represent critically hypoperfused tissue. The region of diffusion-perfusion "mismatch" with normal diffusion but abnormal perfusion is, in theory, the region at risk of 
evolving to infarction if reperfusion treatment is not received. Given the simple attractiveness of the theory and the rather straightforward imaging approaches, DWI/PWI mismatch soon gained wide acceptance as a clinical tool to guide acute stroke therapy. However, skepticism about the validity of the DWI/PWI mismatch theory and its clinical utility have also been raised by many investigators $[17,18]$. Specifically, the observations of the acute reversal of DWI lesions and the failure of all diffusion lesions to evolve to infarction suggest that the presumption of DWI-defined lesions truly reflecting the irreversibly damaged ischemic core may be invalid. Similarly, it has been difficult to define the measurement of perfusion that differentiates tissue that is mildly hypoperfused but not in danger of infarction from tissue that is critically hypoperfused and will go on to die quickly if not reperfused. Whether or not imaging (with the exception of non-contrast $\mathrm{CT}$ at admission) can be employed for management of acute ischemic stroke patients remains to be answered.

In this special issue, several major areas of neuroimaging in the application of acute ischemic stroke are discussed. Davis et al. [19] provide appealing arguments in support of the PWI/DWI mismatch as a potential means for identifying a sub-group of patients who may benefit from tPA beyond $4.5 \mathrm{~h}$ or other reperfusion therapies. Specifically, although DWI signal reversal has been observed, pooled Echoplanar Imaging Thrombolytic Evaluation Trial (EPITHET) and Diffusion and Perfusion Imaging Evaluation for Understanding Stroke Evolution Study (DEFUSE) data suggest that DWI reversal is rare particularly for reperfusion beyond $3 \mathrm{~h}[20,21]$. In addition, without reperfusion, DWI lesions are typically expanded into the PWI-defined abnormal areas $[22,23]$. Finally, reperfusion typically leads to favorable clinical outcomes [22]. Nevertheless, Davis et al. also acknowledge that conclusive evidence from a scientifically rigorous designed phase III trial in support of the DWI/ PWI mismatch hypothesis remains lacking.

Following Davis et al.'s article [19], Powers [24] points out why the past and currently ongoing clinical trials have not provided conclusive evidence to prove or disprove the DWI/PWI hypothesis. Rigorous experimental designs are offered by Powers [24] such that the hypothesis of DWI/ PWI can be addressed once for all. Specifically, a three-arm design is proposed where patients will be randomized into three groups: Group 1 does not receive imaging or treatment; group 2 does not receive imaging, but all are treated; and group 3 receives imaging to guide therapy. In so doing, the trial will not only address the added values of imaging to guide therapy but also account the potential adverse effects of imaging including delay in receiving initial therapy. Nevertheless, as pointed out by Powers, this trial can be difficult to accomplish owing to the required large sample size. An alternative two-step design is then proposed in the article. Powers further goes on to argue that, given the known risk of symptomatic intracranial hemorrhage accompanying reperfusion therapies, their use should be restricted to such well-designed trials.

One of the prerequisites for the utilization of DWI/PWI mismatch or, for that matter, CT perfusion for future clinical trials is the choices of parameters (or threshold values) to define not only the DWI and PWI lesions but also what imaging signatures contribute to mismatch. The outcome of any clinical trial most likely depends on the choice of these parameters. Specifically, although not specifically designed, several clinical trials (DEFUSE [25] and EPITHET [22]) have attempted to determine how to use the DWI/PWI mismatch for identifying a sub-group of patients who may benefit from reperfusion therapies. Results thus far have been inconsistent. In addition to the pitfalls discussed in both Davis et al.'s [19] and Powers's [24] articles, the wide variability of definitions for determining DWI and PWI lesions and what constitutes to mismatch are perhaps the main culprits. For example, DEFUSE [25] used Tmax $>2 \mathrm{~s}$ as PWI lesion, but DEFUSE-2 has changed it to Tmax $>6 \mathrm{~s}$ instead. The definition of mismatch has also been modified from the original $20 \%$ difference between DWI- and PWIdefined lesions in DEFUSE to now PWI/DWI lesion $>1.8$ in DEFUSE-2 [26, 27]. Furthermore, Zhu et al. [28] draw attention to the excellent article by Dani et al. [29] in which the authors reviewed the published definitions of core, penumbra, and normal tissues using either MR or CT perfusion; a total of 4,17 , and 2 different definitions for core, penumbra, and normal tissues have been reported. Together, these reports point out the difficulties of objectively choosing a set of parameters that are biologically justified. To a large extent, this difficulty may not be surprising. Unlike PET where quantitative measures of cerebral hemodynamics are available, only relative measures can be obtained using MRI. In addition, although numerous results have been reported suggesting the potential underlying biological mechanisms leading to the observed diffusion signal changes during acute stroke [30,31], conclusive evidence remains elusive. These inherent limitations associated with MR DWI/PWI may be difficult to overcome. Similar problems are also associated with the utilization of CT perfusion for patient selection. Although CT perfusion appears capable of quantitatively measuring cerebral hemodynamics thanks to the linear relation between tracer concentration and x-ray attenuation, the accuracy of the CT perfusion measures remains elusive [32]. The difficulty of choosing a set of biologically meaningful thresholds also holds for CT perfusion. Zhu et al. [28] extensively discuss this particularly problem and call for standardization for stroke CT perfusion, which is clearly of critical importance to ensure that consistent results can be obtained for any given multicenter studies. For the exact same reasons, standardization 
processes should also be conducted for MR. However, standardization is a complex process, particularly in light of the lack of direct evidence discerning the underlying biological mechanisms of the imaging-derived parameters. With these difficulties, one may have to settle with a data-driven approach utilizing the available images collected from previous trials to derive a set of parameters offering clinical utility in delineating the sub-group of patients for subsequent trials. Nevertheless, one must be cautious that outcomes of any clinical trials using the above-mentioned approach most likely cannot be generalized to different times after onset or different treatments.

Since almost all acute stroke patients will first undergo CT imaging to determine eligibility for tPA, with minimal effort and delay, CT perfusion could be easily obtained from all patients. d' Esterre et al. [32] provide a comprehensive overview of CT perfusion approaches, including data acquisition, mathematical framework for obtaining tissue perfusion, and clinical applications. In addition, the potential clinical utility of CT permeability surface (PS) product is also addressed. In particular, the authors conducted a study to determine if PS measures at admission differ between patients with and without subsequent hemorrhage. With a PS threshold of $0.23 \mathrm{ml} / \mathrm{min} / 100 \mathrm{~g}, 77 \%$ sensitivity and $94 \%$ specificity in predicting subsequent hemorrhage were achieved, suggesting the potential clinical utility of this approach [32].

The lack of underlying biological justification for choosing different threshold values discerning DWI/PWI mismatch (and CT perfusion) is also partially addressed by Ford et al. [33] who propose to directly image the "ischemic penumbra." Specifically, they point out that the measurements of the cerebral metabolic rate of oxygen utilization may allow the identification of ischemic penumbra in vivo. Although PET is capable of providing quantitative measures of the cerebral metabolic rate of oxygen $\left(\mathrm{CMRO}_{2}\right)$, which has been shown in animal models of acute stroke to accurately delineate irreversibly damaged tissue $[34,35]$, the logistics of utilizing PET in an acute setting even in the most experienced hands make this approach impractical. Alternatively, although not identical, MR also shows promise of obtaining a measure that may offer similar information to that of PET $\mathrm{CMRO}_{2}$ [36-39]. An et al. [40] report their experience with MR-derived quantitative oxygen extraction fraction and oxygen metabolic index in normal subjects and the effects of multiple episodes of hypercapnia. Highly reproducible measurements of these parameters that correspond well with published normal values were reported. Nevertheless, this MR parameter has not been clinically validated in a paired comparison; a single-center clinical trial doing so is ongoing.

Ford et al. [33] also address the issue of the best data analysis scheme to allow the determination of imaging threshold values for ischemic core, penumbra, and oligemia. Specifically, unlike the past and current ongoing studies using DWI/PWI where patients are grouped into either with or without the presence of targeted mismatch, Ford et al. [33] suggest a tissue-based analysis approach. Using a threeimaging time point design, at baseline, immediately after tPA, and 1-3 month follow-up, the imaging data could offer a means to separate brain tissues into different categories based on the presence or absence of reperfusion after treatment and the final fate of the tissues (Table 1 in Ford et al.) which in turn allow experimental derivation of imaging threshold for ischemic core, penumbra, and oligemia. This proposed approach is most likely to provide a comprehensive analysis as to whether or not imaging methods can be employed to identify operator-defined ischemic penumbra. Nevertheless, the tissue-level analysis approach will require a large sample size. Furthermore, given the highly dynamic nature of ischemic stroke, only two imaging time points during acute phase may not be sufficient to carefully discern temporal changes of ischemic lesions although this is probably the best that can be accomplished in a clinical setting.

The potential clinical utility of the arterial spin labeling approach in acute stroke is discussed by Zaharchuk [41]. Unlike the dynamic susceptibility contrast (DSC) approach, one of the most widely used approaches for obtaining cerebral hemodynamics, the arterial spin labeling approach does not require the injection of a MR contrast agent, allowing repeated measurements. In addition, since protons are magnetically labeled, one could easily translate MR perfusion results to PET O-15 water studies since both utilize the freely diffusible tracer-water-for the estimation of cerebral hemodynamics. However, the sensitivity to systematic errors, the relatively long acquisition time compared to DSC, and the somewhat lower signal-to-noise ratio have made the arterial spin labeling approach less appealing in an acute setting. Zaharchuk [41] carefully addresses the pitfalls associated with the arterial spin labeling approach and offers potential solutions to mitigate these shortcomings. With the continuing improvement in both imaging methods and hardware, it is highly likely that the arterial spin labeling may become one of the main approaches for providing tissue perfusion during acute stroke.

Finally, Boada et al. [42] discuss the novel approach of $\mathrm{Na}$ imaging which potentially holds great promise for determining tissue viability. The appeal of using $\mathrm{Na}$ imaging stems from the tight interrelation of ischemia and sodiumpotassium-ATPase (Na-K-ATPase). During cerebral ischemia, the reduced oxygen supply leads to the failure of $\mathrm{Na}-$ $\mathrm{K}$-ATPase which in turn causes a local increase of intracellular $\mathrm{Na}$ concentration [43-45]. Despite the biologically sound reasons for $\mathrm{Na}$ imaging in acute stroke, it has not been widely utilized. Boada et al. [42] discussed several potential technically challenging factors as well as the 
solutions to circumvent these difficulties. Highly impressive experimental results obtained from clinically available MR scanners are provided, demonstrating the potential feasibility of $\mathrm{Na}$ imaging for acute stroke.

In summary, although neuroimaging has been widely employed in hyperacute cerebral ischemia, its clinical utility, particularly for the management of acute stroke patients, remains to be proven. Nevertheless, based on the reported results thus far, one could suggest that:

1. Standardization of the imaging protocols to permit comparison of parameters used to define ischemic lesions is needed.

2. A scientifically rigorous clinical trial is needed to prove or disprove the true clinical value of imaging (MR and CT) in the management of acute stroke patients.

3. Tissue-level analysis may be valuable in delineating salvageable brain regions.

If we assume that all of the above-outlined items can be accomplished, three technical requirements should also be further considered, including:

1. Rapid data acquisition since "time is brain"

2. Insensitive to or minimizing motion-related artifacts

3. The availability of rapid and automated data analysis schemes

Finally, given the rapid progress of new and novel imaging technologies and lessons learned from the previous studies, we look forward to the performance of rigorously designed randomized clinical trials that will demonstrate whether neuroimaging other than non-contrast CT should be an integral part of the evaluation of patients with hyperacute ischemic stroke.

\section{References}

1. Powers WJ, et al. Cerebral blood flow and cerebral metabolic rate of oxygen requirements for cerebral function and viability in humans. J Cereb Blood Flow Metab. 1985;5(4):600-8.

2. Baron JC, et al. Noninvasive tomographic study of cerebral blood flow and oxygen metabolism in vivo. Potentials, limitations, and clinical applications in cerebral ischemic disorders. Eur Neurol. 1981;20(3):273-84.

3. Wise RJ, et al. Serial observations on the pathophysiology of acute stroke. The transition from ischaemia to infarction as reflected in regional oxygen extraction. Brain. 1983;106(Pt 1):197-222.

4. Moseley ME, et al. Diffusion-weighted MR imaging of acute stroke: correlation with T2-weighted and magnetic susceptibilityenhanced MR imaging in cats. AJNR Am J Neuroradiol. 1990;11 (3):423-9.

5. Rosen BR, et al. MR depicts perfusion of brain and heart. Diagn Imaging (San Franc). 1991;13(11):105-10.

6. No authors listed. Tissue plasminogen activator for acute ischemic stroke. The National Institute of Neurological Disorders and Stroke rt-PA Stroke Study Group. N Engl J Med. 1995; 333(24): 1581-7.

7. Hacke W, et al. Thrombolysis with alteplase 3 to 4.5 hours after acute ischemic stroke. N Engl J Med. 2008;359(13):1317-29.
8. Lees KR, et al. Time to treatment with intravenous alteplase and outcome in stroke: an updated pooled analysis of ECASS, ATLANTIS, NINDS, and EPITHET trials. Lancet. 2010;375 (9727):1695-703.

9. Paciaroni M, Caso V, Agnelli G. The concept of ischemic penumbra in acute stroke and therapeutic opportunities. Eur Neurol. 2009;61(6):321-30.

10. Heiss WD. The ischemic penumbra: correlates in imaging and implications for treatment of ischemic stroke. The Johann Jacob Wepfer award 2011. Cerebrovasc Dis. 2011;32(4):30720.

11. Smith WS, et al. Mechanical thrombectomy for acute ischemic stroke: final results of the Multi MERCI trial. Stroke. 2008;39 (4):1205-12.

12. Penumbra Pivotal Stroke Trial Investigators. The penumbra pivotal stroke trial: safety and effectiveness of a new generation of mechanical devices for clot removal in intracranial large vessel occlusive disease. Stroke. 2009;40(8):2761-8.

13. Costalat V, et al. Rescue, combined, and stand-alone thrombectomy in the management of large vessel occlusion stroke using the solitaire device: a prospective 50-patient single-center study: timing, safety, and efficacy. Stroke. 2011;42(7):1929-35.

14. Rohl L, et al. Viability thresholds of ischemic penumbra of hyperacute stroke defined by perfusion-weighted MRI and apparent diffusion coefficient. Stroke. 2001;32(5):1140-6.

15. Neumann-Haefelin T, et al. Diffusion- and perfusion-weighted MRI. The DWI/PWI mismatch region in acute stroke. Stroke. 1999;30(8):1591-7.

16. Schwamm LH, et al. Time course of lesion development in patients with acute stroke: serial diffusion- and hemodynamic-weighted magnetic resonance imaging. Stroke. 1998;29(11):2268-76.

17. Kidwell CS, et al. Thrombolytic reversal of acute human cerebral ischemic injury shown by diffusion/perfusion magnetic resonance imaging. Ann Neurol. 2000;47(4):462-9.

18. Fiehler J, et al. Severe ADC decreases do not predict irreversible tissue damage in humans. Stroke. 2002;33(1):79-86.

19. Davis $\mathrm{S}$, et al. Perfusion/diffusion mismatch is valid and should be used for selecting delayed interventions. TSR, 2012.

20. Chemmanam T, et al. Ischemic diffusion lesion reversal is uncommon and rarely alters perfusion-diffusion mismatch. Neurology. 2010;75(12):1040-7.

21. Campbell BC, et al. The infarct core is well represented by the acute diffusion lesion: sustained reversal is infrequent. J Cereb Blood Flow Metab. 2012;32(1):50-6.

22. Davis SM, et al. Effects of alteplase beyond $3 \mathrm{~h}$ after stroke in the Echoplanar Imaging Thrombolytic Evaluation Trial (EPITHET): a placebo-controlled randomised trial. Lancet Neurol. 2008;7 (4):299-309.

23. Barber PA, et al. Prediction of stroke outcome with echoplanar perfusion- and diffusion-weighted MRI. Neurology. 1998;51 (2):418-26.

24. Powers WJ. Perfusion-diffusion mismatch: does it identify who will benefit from reperfusion therapy? TSR, 2012.

25. Albers GW, et al. Magnetic resonance imaging profiles predict clinical response to early reperfusion: the diffusion and perfusion imaging evaluation for understanding stroke evolution (DEFUSE) study. Ann Neurol. 2006;60(5):508-17.

26. Albers G. Results of DEFUSE-2: Imaging endpoints. International Stoke Conference, 2012.

27. Lansberg MG. Results of DEFUSE-2: clinical endpoints. International Stoke Conference, 2012.

28. Zhu GM, et al. Standardization of stroke perfusion CT for reperfusion therapy. TSR, 2012.

29. Dani KA, et al. Computed tomography and magnetic resonance perfusion imaging in ischemic stroke: definitions and thresholds. Ann Neurol. 2011;70(3):384-401. 
30. Harkins KD, et al. Assessment of the effects of cellular tissue properties on ADC measurements by numerical simulation of water diffusion. Magn Reson Med. 2009;62(6):1414-22.

31. Gass A, Niendorf T, Hirsch JG. Acute and chronic changes of the apparent diffusion coefficient in neurological disorders-biophysical mechanisms and possible underlying histopathology. J Neurol Sci. 2001;186 Suppl 1:S15-23.

32. d'Esterre CD, et al. Improving acute stroke management with computed tomography perfusion: a review of imaging basics and applications. TSR, 2012.

33. Ford AL, et al. Defining the ischemic penumbra using hyperacute neuroimaging: deriving quantitative ischemic thresholds. TSR, 2012.

34. Baron JC. Mapping the ischaemic penumbra with PET: a new approach. Brain. 2001;124(Pt 1):2-4.

35. Touzani $\mathrm{O}$, et al. Progressive impairment of brain oxidative metabolism reversed by reperfusion following middle cerebral artery occlusion in anaesthetized baboons. Brain Res. 1997;767(1):1725.

36. An H, Lin W. Quantitative measurements of cerebral blood oxygen saturation using magnetic resonance imaging. J Cereb Blood Flow Metab. 2000;20(8):1225-36.
37. An H, et al. Quantitative measurements of cerebral metabolic rate of oxygen utilization using MRI: a volunteer study. NMR Biomed. 2001;14(7-8):441-7.

38. An H, et al. Evaluation of MR-derived cerebral oxygen metabolic index in experimental hyperoxic hypercapnia, hypoxia, and ischemia. Stroke. 2009;40(6):2165-72.

39. Lee JM, et al. Magnetic resonance cerebral metabolic rate of oxygen utilization in hyperacute stroke patients. Ann Neurol. 2003;53(2):227-32.

40. An H, et al. Quantification of cerebral blood flow, oxygen extraction fraction and oxygen metabolic index in human with inhalation of air and carbogen. TSR, 2012.

41. Zaharchuk G. Arterial spin labeling for acute stroke: practical considerations. TSR, 2012.

42. Boada FE, et al. Sodium MRI and assessment of irreversible tissue damage during hyperacute stroke. TSR, 2012.

43. LaVerde $\mathrm{G}$, et al. Serial triple quantum sodium MRI during non-human primate focal brain ischemia. Magn Reson Med. 2007;57(1):201-5.

44. Jones SC, et al. Stroke onset time using sodium MRI in rat focal cerebral ischemia. Stroke. 2006;37(3):883-8.

45. Boada FE, et al. Loss of cell ion homeostasis and cell viability in the brain: what sodium MRI can tell us. Curr Top Dev Biol. 2005;70:77-101. 